\title{
Factores psicosociales motivacionales y estado de salud
}

\author{
Motivational psychosocial factors \\ and health status
}

\author{
Antonio Joaquín Vázquez Díaz \\ SADIN-Federación (Sindicato Andaluz de Docentes Interinos) \\ Sevilla. España. \\ Dolores Pérez Cano \\ Consejería de Empleo. Centro de Prevención de Riesgos Laborales. Junta \\ de Andalucía Sevilla. España.

\section{Ricardo Manuel Moreno Ramos} \\ Consejería de Empleo. Centro de Prevención de Riesgos Laborales. Junta \\ de Andalucía Sevilla. España. \\ Francisco Arroyo Yanes \\ Consejería de Empleo. Centro de Prevención de Riesgos Laborales. Junta \\ de Andalucía Sevilla. España.

\section{José Manuel Suárez Delgado} \\ Consejería de Empleo. Centro de Prevención de Riesgos Laborales. Junta \\ de Andalucía Sevilla. España.
}

Recibido: 04-01-10

Aceptado: 22-02-10

Correspondencia:

José Manuel Suárez Delgado

c/ José Carreras, 3

41500 Alcalá de Guadaira (Sevilla). España.

Télef: 696664570

E-mail: suarezdim@yahoo.es

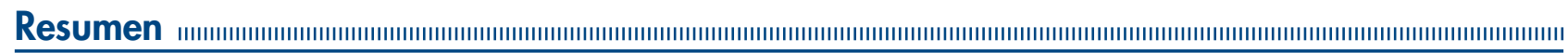

Es conocida la existencia de organizaciones (como las Organizaciones de Servicio Humano (OSH)) caracterizadas por las demandas emocionales y psicosociales que exigen los profesionales que allí trabajan, impactando gravemente en su productividad, calidad de vida y salud mental. Ello también representa consecuencias negativas para los usuarios de dichos servicios. Por lo tanto, resulta necesario identificar factores psicosociales relacionados con el estado de salud mental en profesionales de OSH, objetivo del presente trabajo. Para ello, se evaluaron profesionales Socio-sanitarios (Enfermería, Auxiliares de Clínica) y de la Educación (Técnicos superiores en Educación Infantil, Psicólogos, Profesores, Educadores, Trabajadores Sociales, etc.) de diferentes centros pertenecientes a la Administración Pública de Andalucía.

La evaluación de los factores psicosociales de motivación del trabajador se realizó mediante una escala elaborada "ex profeso". La evaluación del estado de salud mental se realizó con el cuestionario de Goldberg de 28 ítems (GHQ-28). Los resultados mostraron que el 79.2\% de los participantes presentaban más de 7 puntos en la GHQ-28. Entre los factores motivacionales más vinculantes encontramos (por orden de mayor a menor): p46 ("Mi desarrollo profesional me permite conciliar mi vida personal", $r=-0.33$ ), p13 ("Trabajar tiene más ventajas que inconvenientes", $\mathrm{r}=-0.314$ ), p18 ("Me siento partícipe de los éxitos y fracaso de los resultados de mi trabajo", r= -0.282), p25 ("Me siento parte de un equipo"), p32 ("El superior soluciona los problemas de una manera eficaz", $r=-0.269$ ), p26 ("Me resulta fácil expresar mis opiniones en mi lugar de trabajo", r= -0.262). 
Se concluye que las variables psicosociales motivacionales representan factores a tener en cuenta en la definición del estado de Salud en profesionales de OSH.

Med Segur Trab (Internet) 2010; 56 (218): 12-21

Palabras clave: Salud Laboral, Estrés Laboral, Satisfacción Laboral, Motivación Laboral, Enfermedades Ocupacionales.

Abstract

We know the existence of organizations (Human Service Organizations-HSO henceforth) characterized by emotional and psychosocial demands that require professionals who work there, seriously impacting on their productivity, quality of life and mental health. This also represents a negative impact on users of those services. Therefore, it is necessary to identify psychosocial factors associated with mental health professionals HSO, the objective of this work. We evaluated professionals in Health (Nursing, Clinical Assistants) and Education (Senior technicians in Early Childhood Education, Psychologists, Teachers, Educators, Social Workers, etc.) belonging to different centers of Andalusia Public Administration.

The evaluation of psychosocial factors of worker motivation was performed using a scale developed by "ourselves". The assessment of a mental health questionnaire was conducted with the 28-item Goldberg (GHQ-28). The results showed that $79.2 \%$ of the participants had more than 7 points in the GHQ-28. Among the motivational factors found to be more binding (in order of highest to lowest): p46 ( My professional development allows me to reconcile my personal life", $r=-0.33$ ), p13 ("Work has more advantages than disadvantages", $r=-0,314$ ), p18 ( I am privy to the successes and failures of the results of my work", $r=-0,282$ ), p25 ("I feel part of a team), p32 (" The boss solves the problems effectively ", r = -0,269), p26 (" I can easily express my opinions in my workplace “, $\mathrm{r}=-0,262)$.

We conclude that psychosocial variables account for motivational factors to be considered in defining the health status of HSO professionals.

Med Segur Trab (Internet) 2010; 56 (218): 12-21

Key words: Occupational Health, work stress, job satisfaction, work motivation, Occupational Diseases. 


\section{INTRODUCCIÓN}

Es bien sabido que en el pasado existió un amplio dominio del modelo biomédico respecto al concepto de salud, en donde había una fuerte tendencia centrada en la enfermedad; esta última se entendía en términos biológicos que, a su vez, podían tener consecuencias psicológicas, pero lo psicológico no podía derivar en un problema de salud físico. Desde esta perspectiva, la salud se definía como ausencia de enfermedad ${ }^{(1,2)}$.

Sin embargo, ante la aparición de alteraciones funcionales que no podían ser explicados en términos orgánicos, surge la necesidad de dar espacio al análisis de la enfermedad desde el enfoque psicológico(3). De esta forma, nace el concepto de lo "psicosomático". Junto con éste y muchos otros fenómenos científicos y sociales, fue creciendo el enfoque que considera que la salud está determinada no sólo por aspectos biológicos, sino también por aspectos psicológicos y sociales.

La Organización Mundial de la Salud (OMS) define a la Salud como "un estado completo de bienestar físico, mental y social, y no solamente la ausencia de afecciones o enfermedades"(4). Algunos estudios muestran las afectaciones en la salud mental que se propician en diferentes actividades laborales, como es el caso de los empleados técnicos y administrativos ${ }^{(5)}$ y, en especial, las actividades en Organizaciones de Servicio Humano ${ }^{(6)}$.

Las Organizaciones de Servicio Humano (OSH) (lugares de trabajo de profesionales que ofrecen un servicio humano) tienen dos propiedades principales que las distinguen: se trabaja directamente con personas y se protege y promueve su bienestar. Esta concepción de las OSH se aplica a diversas organizaciones, tales como escuelas, agencias de policía, hospitales e institutos, entre otros. Entre las profesiones que trabajan en estas organizaciones se encuentran los trabajadores sociales, maestros, profesores, policías, enfermeras, etc. Todas estas profesiones están caracterizadas por altas exigencias emocionales.

Existen numerosas investigaciones realizadas con estos profesionales en distintos países en donde se demuestra que el contacto continuo con personas y la alta carga afectiva propician una gran afectación en la salud mental de estos trabajadores ${ }^{(7)}$, lo que conlleva consecuencias directas e indirectas, tanto en los mismos profesionales, las OSH, los usuarios y su entorno familiar. Sugieren que existe una serie de factores psicosociales que inciden en la salud mental de los profesionales de OSH, especialmente la escasez de personal (que supone sobrecarga laboral), trabajo en turnos, trato con usuarios problemáticos, falta de especificidad de funciones y tareas, falta de autonomía y autoridad en el trabajo para poder tomar decisiones, falta de confianza en los estamentos superiores, rápidos cambios tecnológicos, diversas variables de carácter demográfico, el tipo de profesión, clima laboral, contenido del puesto y características de personalidad, entre otras.

Por lo tanto, la mayor exigencia a los profesionales de OSH es de carácter psicosocial. Según Maslach ${ }^{(8)}$ existen exigencias psicosociales "universales" que afectan a la salud de los trabajadores de forma crónica, independientemente de las características específicas de la actividad laboral, tales como: la falta de recompensas, las falsas expectativas y, principalmente, la carga de trabajo, el grado de control del trabajador, las relaciones interpersonales y el apoyo social en el trabajo.

$\operatorname{Mohr}^{(9)}$, en un estudio longitudinal, buscó el mejor predictor del estado de salud psicológica en trabajadores manuales y encontró que, de entre varios estresores, la incertidumbre por conservar el empleo fue la variable con mayor peso en esta predicción.

Las relaciones entre factores psicosociales del medio laboral, organización del trabajo, su contenido y su expresión en el deterioro del estado general o la salud psicosomática del individuo han sido estudiadas ampliamente. Las características del puesto de trabajo y el clima organizacional se erigen como causas de burnout y de ciertas manifestaciones psicosomáticas que acontecen en el trabajador ${ }^{(10,11)}$.

Análogamente, el contenido del trabajo, su motivación intrínseca, el diseño de tareas y/o puestos, y la acción humana en el trabajo pueden tener un potencial motivador y contribuyen al bienestar psicológico. Contrariamente, si están deficientemente representadas puede ser una importante fuente de estrés laboral. No obstante, los intentos

Factores psicosociales motivacionales y estado de salud 
de vinculación del burnout con la motivación laboral y el clima organizacional han sido escasos. Asimismo, el clima organizacional puede considerarse, que esencialmente es poco predictor de las psicosomatizaciones, presentándose más bien como pronosticador del burnout. La satisfacción laboral y el burnout tienen una correlación negativa ${ }^{(7)}$.

En el área de la salud laboral el estrés es uno de los problemas más importantes, asociado a un gran número de bajas y de morbilidad, siendo los factores psicosociales (entre ellos la insatisfacción laboral) la fuente más importante de estrés crónico. El estrés es un factor directamente relacionado con el absentismo laboral, llegándose pues, a la conclusión de que la insatisfacción laboral es uno de los factores más importantes en los cuadros de absentismo ${ }^{(12)}$.

Por todo lo anterior, nos ha resultado interesante constatar si una variable psicosocial como es la Motivación laboral, está asociada con la salud mental en profesionales que trabajan en las distintas OSH, en las que nosotros Vigilamos la Salud de sus trabajadores.

\section{MÉTODO}

Se trata de un estudio observacional, no experimental, transversal, descriptivo y correlacional. Participaron 117 profesionales que trabajan en distintas Organizaciones de Servicio Humano (Centros de Atención Socio Educativos, Residencias de tercera edad, Centros de Enseñanza Infantil y Primaria), de las áreas de Enfermería, Auxiliares de clínica, Personal de servicio doméstico, Técnicos de Educación Infantil, Psicología, Trabajo Social y Educación. Fueron divididos los participantes en dos grupos: X (dedicado a la educación) e Y (dedicados a los cuidados de salud). Todos los participantes lo hicieron de forma voluntaria. Se les solicitó consentimiento tras informarlos adecuadamente de los objetivos de nuestro trabajo. Se realizó un muestreo intencional con selección aleatoria de las unidades muestrales últimas (período comprendido entre Enero y Abril de 2009) entre los trabajadores que acudieron a reconocimiento en el Área de Vigilancia de Salud del Centro de Prevención de Riesgos Laborales de nuestra provincia. Solo un $5.98 \%$ (7/117) declinó participar en el estudio.

\section{Hipótesis de trabajo}

Nuestra hipótesis de trabajo consiste en plantear si los factores psicosociales de motivación predicen los niveles de salud mental del grupo de profesionales de Servicios Humanos que hemos estudiado.

\section{Objetivo}

Identificar los mejores predictores psicosociales de salud mental (en referencia a factores motivacionales externos e internos) de un grupo de profesionales de Servicios Humanos de la Administración Pública de Andalucía.

\section{Material}

- Cuestionario de Motivación y Satisfacción desarrollado "ex profeso" por el equipo de psicólogos de nuestro grupo de trabajo. Incluye variables de motivación internas y externas.

- Cuestionario General de Salud Mental (GHQ). Incluye las variables de síntomas somáticos, disfunción social, depresión y problemas de ansiedad-insomnio, en su versión de 28 reactivos (ítems).

Se aplicaron los cuestionarios en una sola vez, en los mismos centros de trabajo donde pertenecían los profesionales en lo referente al grupo X (dedicado a la educación). En lo referente al grupo de profesionales Y (dedicado a los cuidados de salud), la entrevista se realizó en nuestra consulta del Área de Vigilancia de Salud del Centro de Prevención de Riesgos Laborales. La aplicación se realizó de forma individual y anónima. 
Para el análisis de resultados se utilizó la metodología propia de la estadística descriptiva y análisis de varianza, prueba " $t$ " de Student y Chi-cuadrado, para verificar la asociación entre las variables utilizadas. También se utilizaron los coeficientes de correlación lineal de Pearson y de determinación, con el fin de verificar las relaciones entre variables, así como identificar los mejores predictores de Salud Mental.

Se realizó un análisis sobre la fiabilidad y validez del cuestionario de Motivación y Satisfacción Laboral. Para la fiabilidad se utilizó el Coeficiente Alfa de Cronbach. Para el análisis de la validez se empleó la "validez de constructo" mediante la técnica de la Red Nomológica.

\section{RESULTADOS}

En cuanto a las características de la población, el $66.66 \%$ de los participantes pertenecían a tres residencias de tercera edad y 3 guarderías públicas (grupo Y), mientras que el 33.34\% trabajaban en el sector de la educación en centros de educación infantil y primaria (grupo X). El promedio de edad fue de 42.81 años (rango de 20-65), (grupo $\mathrm{X}=37.86$, grupo $\mathrm{Y}=45.28$ ) la mayoría de los participantes era de sexo femenino con un resultado global del $79.48 \%$ (grupo $\mathrm{X}=29.03 \%$, grupo $\mathrm{Y}=56.41 \%$ ) y el $72.64 \%$ era casada. El $69.23 \%$ de los participantes tenía al menos 1 hijo. El nivel de estudios fue Universitario (39.31\%), Bachillerato-ESO (38.47\%), y Primario (17.9\%).

Respecto de la puntuación obtenida en el GHQ-28, los resultados mostraron que el 79.2 \% de la población de cada Consejería presentaba más de 7 puntos, valor a partir del cual un individuo es considerado como "caso positivo". No contestaron este cuestionario el $7.69 \%$ de los individuos, tanto del grupo $\mathrm{X}$ como del grupo $\mathrm{Y}$. Los valores promedio de las puntuaciones de ambos grupos fueron 10.5 puntos para $\mathrm{X}$, y 10.98 para la Y. Las subescalas que más puntuaron fueron la B (ansiedad-insomnio), seguida de la A (síntomas psicosomáticos).

En lo relativo a la posible influencia de las variables sociodemográficas en las puntuaciones del Cuestionario de Salud Mental de Goldberg-28 ítems, los resultados mostraron que no existían diferencias según el grupo de trabajo (X, Y), nivel de estudios, estado civil, o el número de hijos. Tampoco hubo diferencias significativas según la edad de los participantes, la antigüedad en el puesto de trabajo, el carácter funcionarial o temporal del puesto de trabajo.

Por otra parte, en el análisis de la posible correlación entre los factores psicosociales evaluados y la salud mental, se encontró que estaban relacionadas un número importante de variables. Para la identificación de los mejores predictores de salud mental (factores psicosociales con mayor asociación con el estado de salud mental), se realizó un análisis correlacional para cada una de los ítems del cuestionario de Motivación-Satisfacción laboral respecto de la puntuación total en el GHQ-28. Los resultados mostraron una correlación significativa para los ítems p46 ("Mi desarrollo profesional me permite conciliar mi vida personal", $\mathrm{r}=-0.33$ ), p13 ("Trabajar tiene más ventajas que inconvenientes", $\mathrm{r}=-0.314$ ), p18 ("Me siento partícipe de los éxitos y fracaso de los resultados de mi trabajo", r= -0.282), p5.14 ("Las condiciones laborales influyen en mi salud personal", $r=0.286$ ), p5.12 ("Las condiciones ambientales y laborales me impiden acudir a mi trabajo con entusiasmo", r= 0.273), p25 ("Me siento parte de un equipo", $\mathrm{r}=-0.269$ ), p32 ("El superior soluciona los problemas de una manera eficaz", r= -0.269), p26 ("Me resulta fácil expresar mis opiniones en mi lugar de trabajo", $r=-0.262$ ), p41 ("El superior demuestra dominio y conocimiento de sus funciones y del trabajo que desarrollo", r=-0.256) y p33 ("El superior favorece la comunicación y la participación de todos en los asuntos que afectan a mi trabajo", $r=-0.269$ ).

La variable que presentó mayor asociación con la puntuación total de Salud Mental, fue la conciliación de la vida personal con la actividad profesional seguida de la predisposición personal al trabajo, participación en los éxitos y fracasos de los resultados del trabajo desempeñado, poder expresar fácilmente las opiniones y el apoyo por parte de los superiores.

La satisfacción laboral fue medida mostrando los resultados siguientes: $91.45 \%$ (107/117) estaban satisfechos con su trabajo, mientras que el 8.58 \% (10/117) estaban insatisfechos. No obstante, precisamos de un estudio más profundo al respecto para 
afirmar la veracidad de este resultado. Análogamente, la descripción del perfil motivacional de la población estudiada, quedaría para un estudio posterior.

\section{DISCUSIÓN}

\section{Discusión del problema}

Según la $1^{\text {a }}$ Encuesta Andaluza de Condiciones de Trabajo (13), realizada por el Instituto Andaluz de Prevención de Riesgos Laborales, de la Consejería de Empleo de la Junta de Andalucía (21 de octubre de 2008), la mayoría de los trabajadores, el 79,4\%, no perciben consecuencias negativas del trabajo en su salud, mientras que sí lo hace el $18,4 \%$.

Las afecciones de la salud atribuidas al desempeño profesional son, mayoritariamente, de naturaleza musculoesquelética: dolor de espalda (según el 59,8\% de quienes creen que el trabajo afecta a su salud), dolor de cuello o nuca $(32,1 \%)$. El estrés en cualquiera de sus presentaciones, ocuparía un tercer lugar con un $27,2 \%$ de los casos.

Este último se da con más frecuencia en las actividades del Sector Servicios, mientras que la mayor parte de las demás dolencias se manifiestan principalmente en el Sector Primario, Industria y Construcción. Entre el 18,4\% de los trabajadores que consideran que su trabajo afecta a su salud, la mitad ha acudido a consulta médica a causa de esas dolencias.

Algo más de un tercio de la población activa en Andalucía (37,0\%) afirma tener alguna sintomatología psicosomática, achacable al menos en parte a la actividad laboral. La aparición de estos síntomas es más frecuente en los casos de trabajadores sujetos a un nivel de exigencia mental elevado, en particular cuando las tareas son muy repetitivas o de muy corta duración y cuando se trabaja muy rápido o con plazos muy cortos o estrictos. También se presenta más habitualmente esta sintomatología cuando las relaciones personales y el clima laboral son evaluados negativamente.

Los trastornos mentales son la segunda causa de IT de larga duración (14). Uno de cada cinco pacientes relaciona su patología con el trabajo, siendo los diagnósticos más prevalentes los trastornos de adaptación, trastornos mixtos ansioso-depresivos y los episodios depresivos. En tres de cada cuatro casos la duración media de la IT supera los seis meses. Datos similares han sido publicados desde la Comunidad Foral de Navarra (15), respecto del año 2008. No es nuestro objetivo del estudio, pero bien pudiéramos cifrar el coste de esta patología con unas simples reglas de tres.

El estrés laboral es la segunda causa de baja entre el profesorado, por detrás de las enfermedades osteomusculares (16). Afecta en mayor proporción al profesorado de secundaria y se ha convertido en un auténtico problema social. Estadísticas análogas las encontramos en el mundo sanitario. Entre los factores que contribuyen al mismo se han sugerido las reformas educativas, la gran diversidad de alumnado en un mismo centro, la desmotivación y el déficit de atención del alumnado, la presión social y la falta de apoyo de compañeros y equipo directivo.

En todos los ámbitos interviene la motivación como mecanismo para lograr determinados objetivos (17). Aplicada en el ámbito laboral, se puede lograr que los empleados motivados, se esfuercen por tener un mejor desempeño en su trabajo. La motivación es un elemento importante del comportamiento organizacional, que permite canalizar el esfuerzo y la conducta del trabajador, permitiéndole sentirse mejor respecto a lo que hace.

En este mismo orden, el clima organizacional está ligado al grado de motivación de los empleados. Cuando tienen una gran motivación, se establecen relaciones satisfactorias, interés en la colaboración, etc. En sentido inverso, cuando la motivación es escasa, el clima organizacional tiende al desinterés, apatía, descontento, hasta llegar a estados de inconformidad (18-21).

En 1974 Freudenberger (12) relacionó estados de ansiedad y depresión con un estado al que denominó "burnout" ya que la respuesta era la pérdida de ilusión, el absentismo, conductas evitativas y de renuncia, etc. En nuestro trabajo no hemos querido abordar este tema, en tanto que "el burnout" (Síndrome de estar quemado) sería un grado 
máximo de apatía, bien definido en parámetros psicológicos, y que debiera ser estudiado con una metodología específica. Pero no podemos negar, que a esta situación se llega por situaciones de estrés, desmotivación y clima laboral perverso, como está ampliamente reflejado en la bibliografía.

Son las profesiones sanitarias las profesiones que más han acusado este síndrome. Así, los educadores, cuidadores de personas con enfermedades crónicas y degenerativas, los que realizan labores de atención al público, etc serán los más afectados. El pensamiento imperante es la desmotivación, la cual puede desembocar en cuadros que oscilan desde una simple indiferencia hasta cuadros serios de ansiedad, depresión y consumo de drogas.

Para lograr una buena productividad, el trabajador necesita realizar las cosas con satisfacción y contar con los elementos necesarios para desempeñarse adecuadamente (22). Un reto que hoy tienen las empresas es identificar como se sienten sus trabajadores e identificar como ven a la organización de la que forman parte.

En nuestro trabajo se planteó el estudio de tales problemas, en base al hecho de que eran muy numerosos los trabajadores que expresaban su descontento con aspectos variados del trabajo desarrollado. Todos concluían en un punto común: estaban afectos de "ansiedad y estrés", que muchos de ellos lo achacaban directamente a las condiciones laborales. Por ello, nos surgió la necesidad de cuantificar esta problemática, y apreciar cuales eran las causas que la originaban. Bien es cierto, que nuestro cuestionario cubre la mayor parte de los ítems que pueden afectar al aspecto psicosocial del trabajador, pero somos conscientes de que podrían haberse incluido otros.

\section{Discusión del material y método}

David Goldberg desarrolló, en 1972, el cuestionario GHQ (General Health Questionnaire), concebido como método de identificación de pacientes con trastornos psíquicos en el ámbito clínico de los no especialistas en psiquiatría. El más difundido en nuestro país ha sido el GHQ-28, adaptado y, validado por Lobo et al. en 1.985 (23).

El GHQ-28 es un cuestionario autoadministrado que consta de 28 ítems agrupados en cuatro subescalas de 7 ítems cada una: subescala A (síntomas somáticos), subescala B (ansiedad e insomnio), subescala C (disfunción social) y subescala D (depresión grave). Cada pregunta tiene cuatro posibles respuestas, progresivamente peores. Se debe subrayar la respuesta elegida y es esencial que se limite a su situación en las últimas semanas, no en el pasado.

Explora dos tipos de fenómenos: la incapacidad para funcionar, desde el punto de vista psíquico, a nivel "normal" y la aparición de nuevos fenómenos de malestar psíquico. Se trata de un cuestionario breve y de fácil ejecución. Tiene dos aplicaciones principales: estimar la prevalencia de la enfermedad psiquiátrica en una determinada población y captar casos de patología psiquiátrica en las consultas no especializadas. Como elemento de screening tiene ventajas sobre el SCL-90-R, pero como medida de diferentes perfiles psicopatológicos este último ha demostrado su superioridad. Es un buen instrumento para ser utilizado en Atención Primaria y en su versión de 28 preguntas, supone el test más apoyado y consensuado como medio de estudio laboral para detectar signos y síntomas de alteraciones psíquicas menores en la comunidad, tanto para casos ya diagnosticados como ocultos.

El GHQ no sirve para establecer un diagnóstico clínico, aunque las subescalas B (ansiedad/insomnio) y D (depresión grave) se correlacionan bien con la valoración de los psiquiatras para ansiedad y depresión. Sin embargo, esto no ocurre con la subescala A para síntomas somáticos.

El GHQ 28 ítems se utiliza en poblaciones laborales para estudiar relaciones entre salud mental, estrés laboral y trastornos psicosomáticos.

En sus propiedades psicométricas, destacan la fiabilidad y validez. Los datos sobre validez predictiva obtenidos (para un punto de corte de 5/6 [5 no caso /6 caso)] fueron de una sensibilidad ( probabilidad de detectar un + siendo realmente + ) del $84.6 \%$, una especificidad (probabilidad de detectar un - siendo realmente - ) del 82\%, un VPP del $75 \%$, un VPN del $89.3 \%$ y un índice de mal clasificados del 17\%; para un punto de corte

Factores psicosociales motivacionales y estado de salud 
de $6 / 7$ una sensibilidad del 76.9\%, especificidad del 90.2\%, VPP del 83.3\%, VPN del 85.9\% e índice de mal clasificados del $15 \%$.

El cuestionario TECLA (dedicado a la investigación del Clima Laboral) y el CMT (dedicado a la Motivación el Trabajo) de Toro, fueron los que inicialmente planteamos para ser utilizados, pero no se ajustaban a los objetivos de nuestro estudio. Por ello, marcamos unos objetivos concretos y elaboramos un cuestionario "ex profeso" para el estudio, el cual fue debidamente ajustado por el equipo de psicólogos.

Nuestra escala consta de 48 items, divididos en 5 subescalas: concepto del trabajo desempeñado, relación con los compañeros de trabajo, opinión sobre la dirección de la empresa, condiciones ambientales y cuáles serían las causas para abandonar mi trabajo. Algunas de estas variables fueron evaluadas en monoítem.

Se realizó un estudio sobre la fiabilidad y validez de nuestro cuestionario. La fiabilidad representa la consistencia interna de la medida. Para determinarla se usó el Coeficiente alfa de Cronbach, resultando ser de 0.8845 . Se considera que la consistencia interna de un cuestionario es muy buena cuando dicho coeficiente asume valores mayores a 0.8 .

La validez es el grado en que una situación o instrumento de medida mide lo que realmente pretende o quiere medir. En ocasiones, la validez es la "exactitud" de la prueba. Es el criterio fundamental para valorar si el resultado obtenido en un estudio es el adecuado. La validez de constructo (de concepto) se utiliza sobre todo para medidas y variables psicosociales, y mide hasta qué punto el indicador o la definición operativa mide el concepto. El análisis se realizó mediante la técnica de la Red Nomológica.

\section{Discusión de los resultados}

Es interesante comentar que los resultados del GHQ-28 mostraron que el $79.2 \%$ de la población de cada Consejería presentaba más de 7 puntos, valor a partir del cual un individuo es considerado como "caso positivo". Los valores promedio de las puntuaciones de ambos grupos X e Y fueron 10.5 y 10.98 respectivamente. Las subescalas que más puntuaron fueron la B (ansiedad-insomnio), seguida de la A (síntomas psicosomáticos).

Como se observa en los resultados, se encontraron asociaciones estadísticamente significativas entre las puntuaciones obtenidas en la escala de salud mental y variables psicosociales laborales estudiadas. Derivado de ello, se aprecia la importancia que ofrece el colectivo a la conciliación del trabajo con la vida personal, el sentido que tiene el sentirse partícipe de un grupo de trabajo y el valor que ofrece este colectivo al papel desempeñado por los superiores en la solución de problemas. Esto pudiera significar que aquellos profesionales que se encuentran en una situación de escasa disponibilidad temporal para el desarrollo de actividades personales extralaborales, o que no se sienten partícipes en el desempeño del trabajo diario, o el superior no soluciona adecuadamente la problemática común, poseen niveles de hostilidad e irritabilidad elevados y son más vulnerables a la afectación de su salud mental.

Resulta interesante el poder comentar que en este grupo no resultó existir correlación entre la variable económica, la temporalidad e inestabilidad del puesto de trabajo, el carácter funcionarial, el nivel de estudios o el hecho de estar casado con hijos, y la existencia de un resultado positivo en el GHQ-28. Esto confirma la importancia de estas variables motivacionales y su consideración "específica y concreta" en la elaboración de programas de promoción, prevención y vigilancia de salud en Organizaciones de Servicio Humano.

Se demostró que la disponibilidad de tiempo para actividades personales, fue el estresor más nocivo para la salud psicológica en comparación con las variables del modelo de Karasek (24). Tal hecho, implica que probablemente el contexto cultural y de género influya en la nocividad de ciertas variables. Queda claro, asimismo, el papel primordial de la consideración hacia los superiores como predictor estadístico relevante de los niveles de salud mental. 


\section{CONCLUSIONES}

- Es importante realizar exámenes de salud en estos colectivos con la finalidad de detectar y prevenir problemas de salud de naturaleza psicosocial.

- El elemento predictor más potente de alteración del estado de salud mental, en nuestro estudio, resultó ser la imposibilidad de conciliar la vida personal con la actividad profesional. En un segundo lugar se situó la implicación en el trabajo.

- Los superiores o responsables de los centros de trabajo son considerados como factor motivador.

— La motivación económica no resultó ser predictora.

\section{AGRADECIMIENTOS}

Por su colaboración inestimable, agradecemos su participación a María Luisa García Sánchez, Margarita Blanco, Wilfrido Gennes Ochoa, José Antonio Millán Herrera y Ricardo Muñoz Luque.

\section{BIBLIOGRAFÍA}

1. Juárez A. Factores Psicosociales Relacionados con la Salud Mental en Profesionales de Servicios Humanos en México. Cienc Trab. 2004; 6 (14):189-196.

2. León R. Introducción a la Psicología de la salud desde un enfoque psicosocial. Asesoramiento al personal sanitario. Sevilla: Universidad de Sevilla, 2003:133-157.

3. Freud S. La interpretación de los sueños, 1. Madrid: Alianza Editorial, Biblioteca Freud, 2000: 200-249.

4. Cook R. 1993. El derecho internacional y la salud en la mujer. Publicación científica $\mathrm{N}^{\circ} \mathbf{5 4 2}$. Organización Panamericana de la Salud. México: OPS.

5. Gutiérrez R. 2001. Calidad de vida y estrés en ámbitos organizacionales. Rev Mex Psico; 18 (1):182-183.

6. Arita W, Arauz C. 2001. Modelo para la Investigación del Proceso del Síndrome del Burnout. Psic y Sal; 11 (1):75-80.

7. Gil-Monte P, Peiró JM. 1997. Desgaste psíquico en el trabajo: el síndrome de quemarse. Madrid: Síntesis.

8. Maslach C. 2004. The relation between work environment and burnout. In, proceedings from the forum "The way we work and its impact in our health", UCLA, California USA.

9. Mohr, G. 2000. The changing significance of different stressors after the announcement of bankruptcy: a longitudinal investigation with special emphasis on job insecurity. J Org Behav; 21:337-359.

10. Topa Cantisano G, Morales Domínguez J. Determinantes específicos de la satisfacción laboral, el burnout y sus consecuencias para la salud: un estudio exploratorio con funcionarios de presiones. International Journal of Psychology and Psychological Therapy, 2005; 5(1): 73-83.

11. Boada Grau J, Diego Vallejo R, Agulló Tomás E. El burnout y las manifestaciones psicosomáticas como consecuentes del clima organizacional y de la motivación laboral. Psicothema, 2004; 16 (1): 125-131.

12. Castellano Arroyo M, Díaz Franco JJ. Estrés Laboral. Burnout. Trabajo a turnos. Acoso en el trabajo. Suicidio y trabajo. En: Gil Hernández F. Tratado de Medicina del Trabajo. $1^{\mathrm{a}}$ ed. Barcelona: Masson, 2005.

13. Moreno Hurtado J, et al. $1^{\text {a }}$ Encuesta Andaluza de Condiciones de Trabajo. Instituto Andaluz de Prevención de Riesgos Laborales. Consejería de Empleo de la Junta de Andalucía. 21 de octubre de 2008.

14. Rodríguez Ortiz, B. Sintomatología depresiva asociada al trabajo como causa de incapacidad temporal en la Comunidad de Madrid. MAPFRE Medicina, 2005; 16(3):184-194.

15. Instituto Navarro de Salud Laboral ( http://www.cfnavarra.es/insl)

16. Galdeano H, Godoy P, Cruz I. Factores de riesgo psicosocial en profesores de educación secundaria. Arch Prev Riesgos Labor, 2007; 10 (4): 174-180.

17. Huamani Supo LB. Influencia de los factores de motivación en el desempeño laboral. En "www. monografías.com"

18. López Araujo B, Osca Segovia A, Peiró JM. El papel modulador de la implicación con el trabajo en la relación entre el estrés y la satisfacción laboral. Psicothema, 2007; 19(1): 81-87.

19. Tarist T, Cox T, Tisserand M. Engagement at work: an emerging concept. Work and Stress, 2008; 22(3): 185-186.

20. Simpson MR. Angagement at work: a review of the literature. Intenational Journal of Nursing studies, 2008; In press.

21. Munch-Hansen T, et al. Sickness absence and workplace levels of satisfaction with psychologycal work conditions at public service workplaces. Am J Ind Med, 2009; 52: 153-161.

22. Pérez Vera C, Garza Carranza T. Comportamiento organizacional y satisfacción laboral. Revista Panorama Administrativo, 2007; 3: 99-108.

23. Lobo A et al. Validity of the versión of the General Health Questionnaire (GHQ-28) in a Spanish population. Psichological Medicine. 1986; 16; 135-140.

24. Karasek R, Theorell T. Healthy work. Stress, productivity and the reconstruction of working life. New York: Basic Books, 1990. 
Tabla I - Datos poblacionales (I)

\begin{tabular}{lccc}
\hline & Grupo Y & Grupo X & Total/Media \\
\hline Muestra & $78(66.66 \%)$ & $39(33.33 \%)$ & $117(100 \%)$ \\
Edad (años) & $45.28 \pm 8.51$ & $37.86 \pm 9.91$ & $\begin{array}{c}42.81 \pm 9.6 \\
\text { Rango: } 20-65 \text { años. } \\
\text { Mujer: } 79.48 \%\end{array}$ \\
Sexo (Hombre-Mujer) & $12-66$ & $12-27$ & Hombre: $20.52 \%$ \\
$\begin{array}{l}\text { Estado Civil } \\
\text { (Casados-no Casados) }\end{array}$ & $72-16$ & $21-18$ & $\begin{array}{c}\text { Casados: } 79.68 \% \\
\text { No Casados: } 20.32 \% \\
\text { Hijos }\end{array}$ \\
\hline
\end{tabular}

Tabla II - Datos poblacionales (II)

\begin{tabular}{|c|c|c|c|}
\hline & Grupo Y & Grupo $\mathbf{X}$ & Total/Media (\%) \\
\hline Nivel estudio & $\begin{array}{c}\text { Universitario: } 12 \\
\text { Bachillerato-ESO: } 40 \\
\text { Primario: } 24\end{array}$ & $\begin{array}{c}\text { Universitario: } 34 \\
\text { Bachillerato-ESO: } 5 \\
\text { Primario: } 0\end{array}$ & $\begin{array}{c}\text { Universitario: } 39.1 \\
\text { Bachillerato-ESO: } 38.47 \\
\text { Primario: } 17.9\end{array}$ \\
\hline Condición laboral & $\begin{array}{c}\text { Laboral: } 88 \\
\text { Funcionario: } 0\end{array}$ & $\begin{array}{c}\text { Laboral: } 4 \\
\text { Funcionario:35 }\end{array}$ & \\
\hline Contrato laboral & $\begin{array}{c}\text { Eventual-Interino: } 19 \\
\text { Fijo:59 }\end{array}$ & $\begin{array}{c}\text { Eventual-Interino: } 11 \\
\text { Fijo: } 27\end{array}$ & \\
\hline Grupo laboral & $\begin{array}{l}\text { A: } 0 \\
\text { B: } 6 \\
\text { C: } 32 \\
\text { D: } 11 \\
\text { E: } 24\end{array}$ & $\begin{array}{c}\text { A: } 25 \\
\text { B: } 10 \\
\text { C: } 1 \\
\text { D: } 1 \\
\text { E: } 2\end{array}$ & \\
\hline Años vida laboral & $17.30 \pm 8.68$ & $11.53 \pm 9.24$ & \\
\hline Años actual puesto & $11.87 \pm 9.57$ & $6.35 \pm 6.57$ & \\
\hline
\end{tabular}

Tabla III - Puntuaciones del Cuestionario de Salud de Goldberg (GHQ)

\begin{tabular}{cccc}
\hline & Grupo X & Grupo Y & Total \\
\hline A (Somatizaciones) & 3.61 & 3.97 & 3.73 \\
B (Ansiedad-insomnio) & 4.52 & 4.16 & 4.40 \\
C (Disfunción social) & 1.68 & 1.41 & 1.59 \\
D (Depresión) & 1.16 & 0.97 & 1.10 \\
\hline Total & $10.98 \pm 5.77$ & $10.50 \pm 4.97$ & $10.82 \pm 5.77$ \\
$\leq 6$ puntos & $16 / 78$ & $8 / 39$ & $20.8 \%$ \\
$>7$ puntos & $62 / 78$ & $31 / 39$ & $79.2 \%$ \\
\hline
\end{tabular}

